Kroon, D., Norris, R.D., and Klaus, A. (Eds.)

Proceedings of the Ocean Drilling Program, Scientific Results Volume 171B

\section{DATA Report: High-ResOlUtion STABLE ISOTOPE STRATIGRAPHY OF THE LATE MidDle EOCENE At Site 1051, Blake Nose'}

\author{
Bridget S. Wade, ${ }^{2}$ Richard D. Norris, ${ }^{3}$ and Dick Kroon ${ }^{2}$
}

\begin{abstract}
The primary aim of the this investigation was to examine the stability of subtropical sea-surface temperatures and reconstruct the surfaceto-benthos thermal gradient. High-resolution stable isotopic analyses $\left(\delta^{18} \mathrm{O}\right.$ and $\left.\delta^{13} \mathrm{C}\right)$ were conducted on late middle Eocene planktonic and benthic foraminifers recovered from Hole 1051B, Blake Nose, western North Atlantic. The sequence comprises a siliceous nannofossil and foraminifer ooze, with well-preserved calcareous microfossils. Isotopic examination was conducted on the mixed-layer dweller Morozovella spinulosa and the benthic foraminifer Nuttalides truempyi at this subtropical site.
\end{abstract}

\section{INTRODUCTION}

Site $1051\left(30^{\circ} 03^{\prime} \mathrm{N}, 76^{\circ} 21^{\prime} \mathrm{W}\right)$ is located at a water depth of $1980 \mathrm{~m}$ at Blake Nose, western North Atlantic. This site includes an expanded and virtually entire Paleogene section of pelagic and hemipelagic sediments, from Paleocene to early late Eocene age. The sediments consist predominantly of a siliceous nannofossil and foraminifer ooze (Norris, Kroon, Klaus, et al., 1998). The middle Eocene foraminifers recovered at this site are highly suitable for isotopic examination as they are well preserved. In this data report we detail the isotopic analyses conducted on
${ }^{1}$ Wade, B.S., Norris, R.D., and Kroon, D., 2000. Data report: High-resolution stable isotope stratigraphy of the late middle Eocene at Site 1051, Blake

Nose. In Kroon, D., Norris, R.D., and Klaus, A. (Eds.), Proc. ODP, Sci. Results, 171B, 1-14 [Online]. Available from World Wide Web: <http://www-odp. tamu.edu/publications/171B_SR/ VOLUME/CHAPTERS/SR171B05.PDF>. [Cited YYYY-MM-DD]

${ }^{2}$ Department of Geology and

Geophysics, University of Edinburgh, Grant Institute, West Mains Road, Edinburgh, EH9 3JW, Scotland, United Kingdom. Correspondence author: B.Wade@glg.ed.ac.uk

${ }^{3}$ Department of Geology and Geophysics, Woods Hole

Oceanographic Institution, MS-23, Woods Hole MA 02543-1541, USA.

Initial receipt: 14 June 1999

Acceptance: 13 March 2000

Publication: 31 August 2000

Ms 171B-119 
B.S. WADE ET AL.

planktonic and benthic foraminifers from the late middle Eocene of Hole 1051B.

Whereas the climatic shifts during the Eocene are understood in general terms, many specific questions remain unanswered, particularly in terms of the variability of climate, the timing of oceanic cooling, and the effect on oceanographic structure. This is because high-resolution, low-latitude Paleogene records are scarce and previously acquired Eocene records are either incomplete, intermittently cored, or disturbed by drilling through Eocene chert.

Tropical sea-surface temperatures (SSTs) have been shown to be essential to verify the character of forcing and feedback of warm climatic states. However, a disparity prevails between proxy temperature interpretations from isotopic (e.g., Boersma et al., 1987; Zachos et al., 1994; Bralower et al., 1995) and paleontological indices for the middle Eocene tropics (e.g., Adams et al., 1990; Graham, 1994).

We have produced $\delta^{18} \mathrm{O}$ and $\delta^{13} \mathrm{C}$ profiles from well-preserved planktonic foraminifers, which will be used to obtain late middle Eocene SSTs from the subtropical Atlantic. The stable isotope record from Site 1051 will be applied to document and assess the scale and timing of relatively short term climatic changes associated with the switch from the early Eocene greenhouse to the late Eocene icehouse world in the Atlantic Ocean. Benthic foraminifers are analyzed to compare benthic temperatures with nearby Ocean Drilling Program Site 1052 and to obtain a direct measurement of thermal gradients in the intermediate waters over the Blake Nose. In addition, the comparison of temperature records derived from planktonic and benthic foraminifers permits reconstruction of the surface-to-benthos thermal gradient in the western North Atlantic.

\section{METHODS AND PROCEDURES}

For isotopic analyses, $2.8-\mathrm{cm}^{3}$ samples from Hole 1051B were examined at $10-\mathrm{cm}$ intervals. All samples were dried, weighed, soaked in a Calgon/peroxide solution overnight, and wet sieved on a $63-\mu \mathrm{m}$ mesh. The $>63-\mu \mathrm{m}$ size fraction was then oven dried at $<50^{\circ} \mathrm{C}$ and weighed again to obtain a measurement of percentage coarse fraction $(>63 \mu \mathrm{m})$. For planktonic foraminiferal analyses the $>63-\mu \mathrm{m}$ fraction was dry sieved into three different size fractions: $>355 \mu \mathrm{m}, 250-355 \mu \mathrm{m}$, and $<250 \mu \mathrm{m}$. Isotopic examination was conducted on planktonic foraminifers from the 250 - to $355-\mu \mathrm{m}$ size fraction. A narrow size fraction was selected to overcome vital and ontogenetic effects on stable isotopic interpretation (Shackleton et al., 1985; Corfield and Cartlidge, 1991; Pearson et al., 1993).

The mixed-layer dweller Morozovella spinulosa was selected for analysis because of its ease of identification, abundance within the samples, and light $\delta^{18} \mathrm{O}$ values. When this taxon was rare, Morozovella crassata, Acarinia praetopilensis, or Globigerinatheka mexicana were chosen as the best alternatives.

For planktonic isotopic investigation, multiple specimens of the same species were picked for each sample studied, normally 5-20 individuals depending on abundance. Analysis of multiple specimens provides results that are nearer to the species mean stable isotopic value than analyses conducted on single specimens. However, using this method, data on intraspecific deviation are lost (Pearson and Shackleton, 1995). Sample weights were usually $0.17 \pm 0.03 \mathrm{mg}$. Before analy- 
sis, specimens were placed in methanol and cleaned ultrasonically to dislodge attached fine calcite particles. Ultrasonic cleaning was repeated when visual examination proved this to be required.

All planktonic foraminifer samples were analyzed isotopically using a VG Isogas Prism III mass spectrometer at the University of Edinburgh, Scotland. Normal corrections were employed, and results of stable isotope measurements are expressed in parts per thousand relative to the Peedee belemnite (PDB) standard reference carbonate of zero (Craig, 1957). Silver Mine (SM) calcite powdered standard was measured concurrently (mean $=0.20 \mathrm{mg}$ ) to record analytical precision and instrument calibration. Replicate analyses of standards gave rise to standard deviations of $0.09 \%$ for $\delta^{18} \mathrm{O}$ and $0.05 \%$ for $\delta^{13} \mathrm{C}$.

The benthic foraminifer Nuttalides truempyi was picked from the $>150-\mu \mathrm{m}$ fraction and analyzed isotopically with a Finnigan MAT252 mass spectrometer and associated automated carbonate device ("Kiel Device") in the Department of Geology and Geophysics at the Woods Hole Oceanographic Institution. Samples (average weight $=60 \mu \mathrm{g}$ ) were lightly crushed in the reaction vessels to ensure complete reaction with phosphoric acid at $70^{\circ} \mathrm{C}$. Six standards were run with each set of 40 unknowns; standards included Carrara Marble, Atlantis II deep-sea coral, and B-1 marine carbonate. Results were corrected to VPDB (PDB estimated by analysis of NBS-19) followed by a second correction for sample gas volume. Replicate analyses of all three standards yield standard errors of $0.08 \%$ for $\delta^{18} \mathrm{O}$ and $0.04 \%$ for $\delta^{13} \mathrm{C}$ as averages for reproducibility on the A and B lines in the Kiel Device.

Leg 171B material has never been deeply buried, resulting in the excellent preservation of foraminifers. Light and scanning electron microscopy shows the foraminifers to be devoid of carbonate infilling and visible dissolution. Pores on the outer and inner test walls are plainly visible with no surficial overgrowth. Preservation of primary calcite is shown by cross sections through the test walls where pores are open and smooth. A minor amount of fine carbonate debris (mainly coccoliths) are seen attached to the test surfaces. Good preservation is also confirmed by the difference in $\delta^{18} \mathrm{O}$ between the planktonic species and between planktonic and benthic species, whereas uniform $\delta^{18} \mathrm{O}$ values are forecast from models of bulk carbonate diagenetic alteration (Killingley, 1983; Schrag et al., 1995). Our results will contribute to the debate on mechanisms of climate change by establishing subtropical SSTs from well-preserved and complete material, without the consequences of diagenic alteration.

\section{RESULTS}

The data are displayed in Table T1. Here we list sample, depth in meters below seafloor (mbsf), taxon, size fraction, stable isotopic results of oxygen and carbon, and for planktonic foraminifers the number $(N)$ of specimens analyzed.

Warm climatic intervals were previously thought to be periods of relative stability. However, our results reveal large shifts in the $\delta^{18} \mathrm{O}$ values of the subtropical surface waters between $-2 \%$ and 0\%o PDB (Fig. F1). The $\delta^{18} \mathrm{O}$ values obtained from the benthic foraminifers show fluctuations between $0 \%$ and $1.5 \%$ PDB. Large variations are also seen in the planktonic foraminifer $\delta^{13} \mathrm{C}$ values, between $0.7 \%$ and $3.1 \%$ PDB, whereas the $\delta^{13} \mathrm{C}$ values of benthic foraminifer range from $0.2 \%$ to $1.3 \%$ PDB (Fig. F2). The results from Leg 171B will document how rap-
T1. Stable isotope analyses, Hole 1051B, p. 8.

F1. Planktonic and benthic foraminifer $\delta^{18} \mathrm{O}$ record, Hole $1051 \mathrm{~B}$, p. 6.

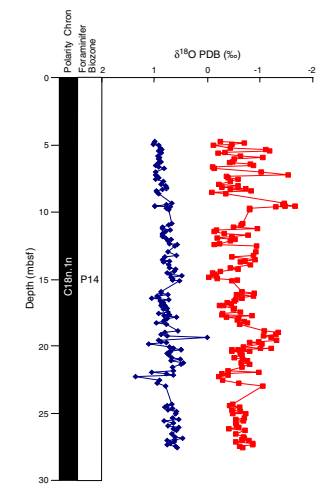

F2. Planktonic and benthic foraminifer $\delta^{13} \mathrm{C}$ record, Hole $1051 \mathrm{~B}$, p. 7.

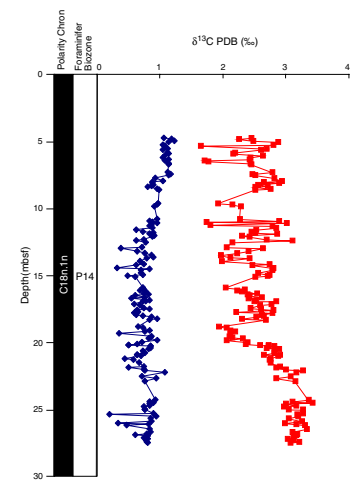


B.S. WADE ET AL.

DATA Report: ISOTOPE STRATIgRAPHy, LATE MidDle EOCENE

4

idly these changes took place and explore the stability of warm climatic phases. The data reported here are discussed and interpreted in Wade et al. (in press).

ACKNOWLEDGMENTS

We would like to thank Colin Chilcott for assistance with the mass spectrometer and Paul Pearson for useful discussions on Eocene planktonic foraminifer taxonomy. This research was supported by UK Natural Environment Research Council reference number GT 04/97/93/ES. Analyses at Woods Hole Oceanographic Institution and sample preparaion were supported by a grant from JOI/USSSP to R.D.N. Susan Drew helped with picking benthic foraminifers and with their analysis in the Woods Hole Oceanographic Institution's mass spectrometry facility. 


\section{REFERENCES}

Adams, C.G., Lee, D.E., and Rosen, B.R., 1990. Conflicting isotopic and biotic evidence for tropical sea-surface temperatures during the Tertiary. Palaeogeogr., Palaeoclimatol., Palaeoecol., 77:289-313.

Boersma, A., Premoli Silva, I., and Shackleton, N.J., 1987. Atlantic Eocene planktonic foraminiferal paleohydrographic indicators and stable isotope paleoceanography. Paleoceanography, 2:287-331.

Bralower, T.J., Zachos, J.C., Thomas, E., Parrow, M., Paull, C.K., Kelly, D.C., Premoli Silva, I., Sliter, W.V., and Lohmann, K.C., 1995. Late Paleocene to Eocene paleoceanography of the equatorial Pacific Ocean: stable isotopes recorded at Ocean Drilling Program Site 865, Allison Guyot. Paleoceanography, 10:841-865.

Corfield, R.M., and Cartlidge, J.E., 1991. Isotopic evidence for the depth stratification of fossil and Recent Globigerinina: a review. Hist. Biol., 5:37-63.

Craig, H., 1957. Isotopic standards for carbon and oxygen and correction factors for mass-spectrometric analysis of carbon dioxide. Geochim. Cosmochim. Acta, 12:133149.

Graham, A., 1994. Neotropical Eocene coastal floras and ${ }^{18} \mathrm{O} /{ }^{16} \mathrm{O}$-estimated warmer vs. cooler equatorial waters. Am. J. Bot., 81:301-306.

Killingley, J.S., 1983. Effects of diagenetic recrystallization on ${ }^{18} \mathrm{O} /{ }^{16} \mathrm{O}$ values of deepsea sediments. Nature, 301:594-597.

Norris, R.D., Kroon, D., Klaus, A., et al., 1998. Proc. ODP, Init. Repts., 171B: College Station, TX (Ocean Drilling Program).

Pearson, P.N., and Shackleton, N.J., 1995. Neogene multispecies planktonic foraminifer stable isotope record, Site 871, Limalok Guyot. In Haggerty, J.A., Premoli Silva, I., Rack, F., and McNutt, M.K. (Eds.), Proc. ODP, Sci. Results, 144: College Station, TX (Ocean Drilling Program), 401-410.

Pearson, P.N., Shackleton, N.J., and Hall, M.A., 1993. Stable isotope paleoecology of middle Eocene planktonic foraminifers and multi-species isotope stratigraphy, DSDP Site 523, South Atlantic. J. Foraminiferal Res., 23:123-140.

Schrag, D.P., DePaolo, D.J., and Richter, F.M., 1995. Reconstructing past sea surface temperatures: correcting for diagenesis of bulk marine carbonate. Geochim. Cosmochim. Acta, 59:2265-2278.

Shackleton, N.J., Corfield, R.M., and Hall, M.A., 1985. Stable isotope data and the ontogeny of Paleocene planktonic foraminifers. J. Foraminiferal Res., 15:321-336.

Wade, B.S., Kroon, D., and Norris, R.D., in press. Orbitally forced climate change in the late middle Eocene at Blake Nose (Leg 171B): evidence from stable isotopes in foraminifera. In Kroon, D., Norris, R.D., and Klaus, A. (Eds.), Western North Atlantic Palaeogene and Cretaceous palaeoceanography. Geol. Soc. Spec. Publ. London.

Zachos, J.C., Stott, L.D., and Lohmann, K.C., 1994. Evolution of early Cenozoic marine temperatures. Paleoceanography, 9:353-387. 
B.S. WADE ET AL.

DAtA Report: Isotope Stratigraphy, LATE MIDDle EOCENE

Figure F1. Middle Eocene planktonic and benthic foraminifer $\delta^{18} \mathrm{O}$ (parts per thousand relative to the Peedee belemnite $[\mathrm{PDB}]$ ) record from Hole 1051B. Squares = Morozovella spinulosa; diamonds = Nuttalides truempyi.

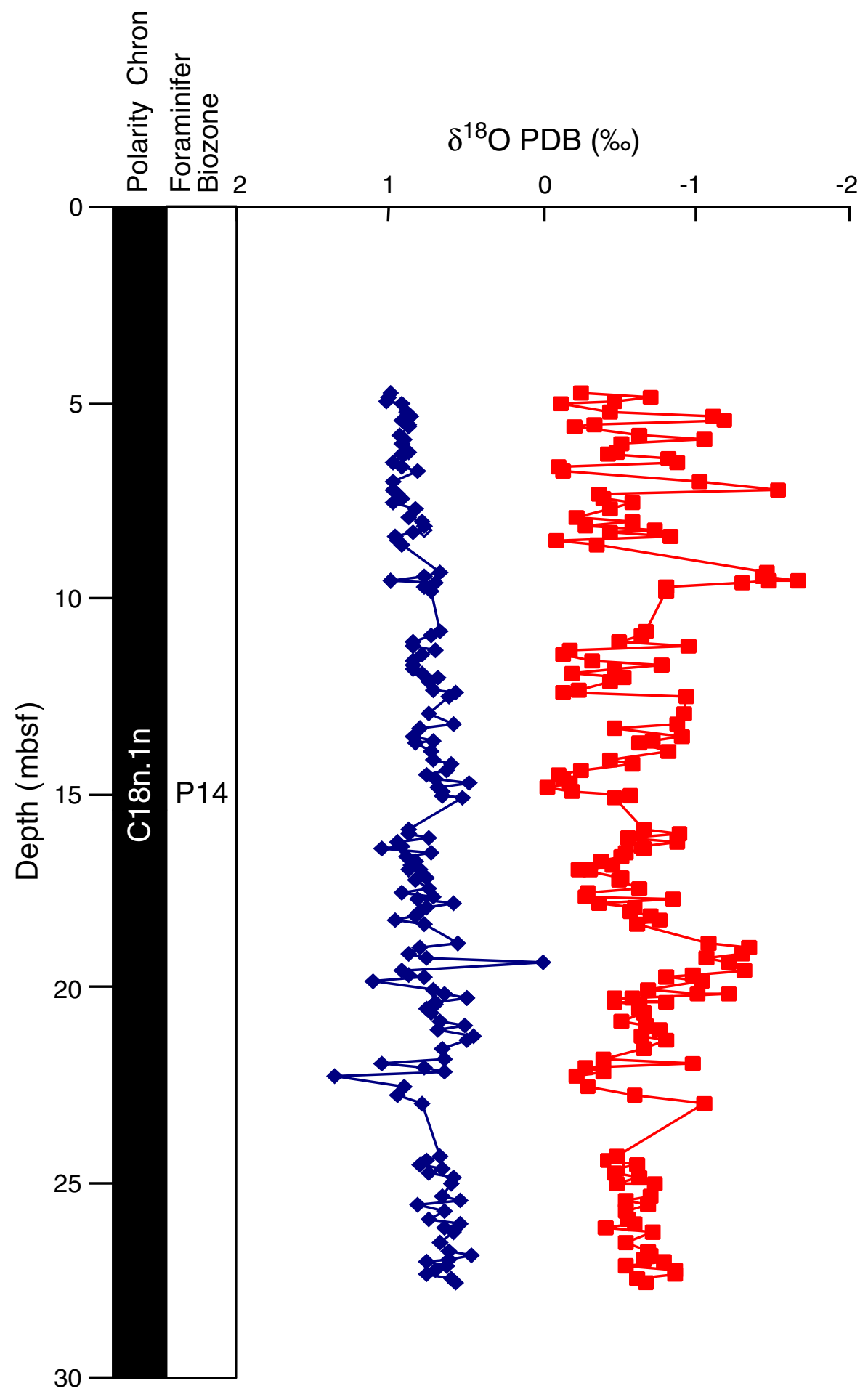


B.S. WADE ET AL.

DAtA Report: Isotope Stratigraphy, LATE MIDDle EOCENE

Figure F2. Middle Eocene planktonic and benthic foraminifer $\delta^{13} \mathrm{C}$ (parts per thousand relative to the Peedee belemnite $[\mathrm{PDB}]$ ) record from Hole 1051B. Squares = Morozovella spinulosa; diamonds = Nuttalides truempyi.

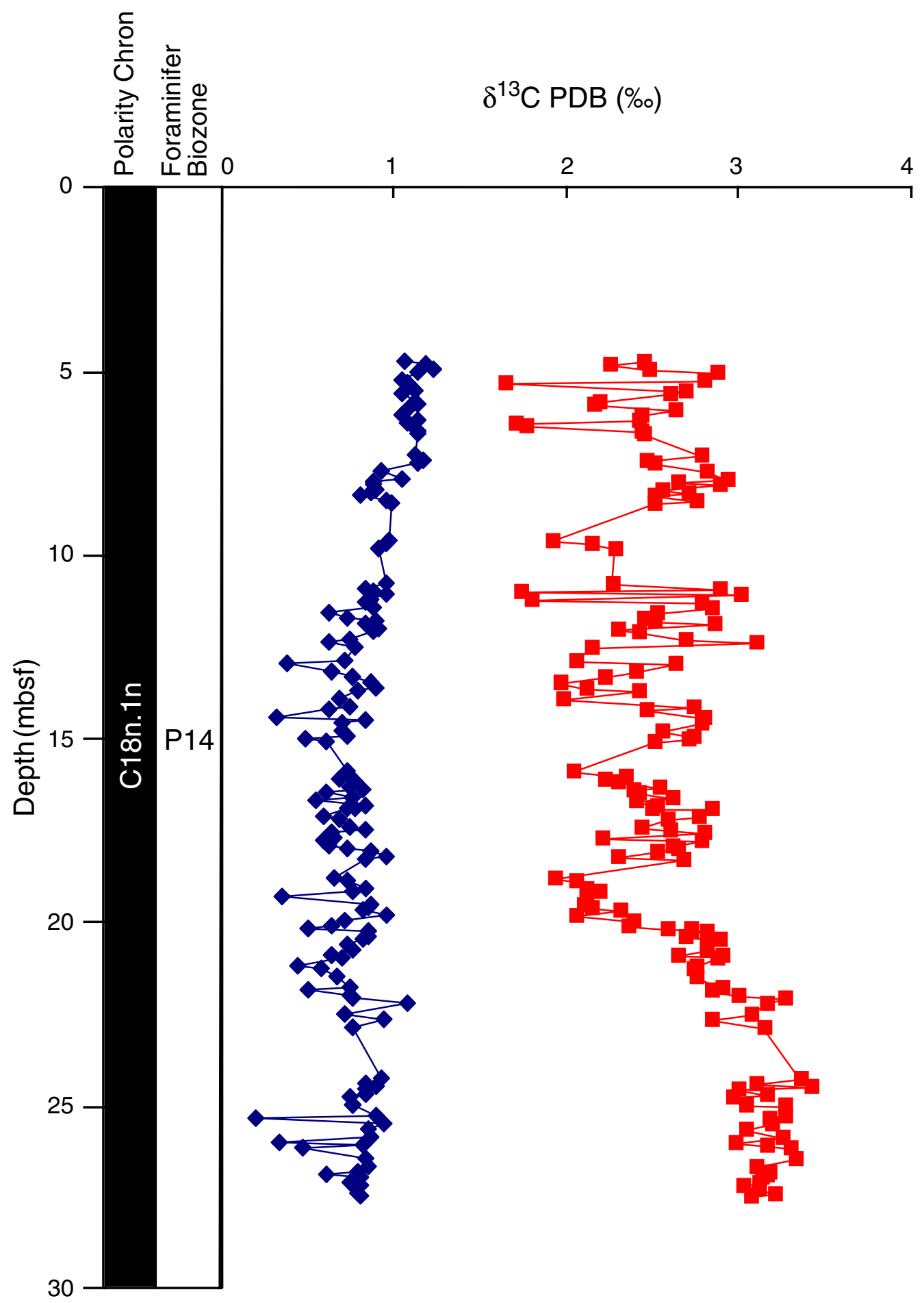


B.S. WADE ET AL.

DATA Report: Isotope Stratigraphy, LATE MidDle EOCENE

Table T1. Results of stable isotope analyses, Hole 1051B. (See table note. Continued on next six pages).

\begin{tabular}{|c|c|c|c|c|c|c|}
\hline $\begin{array}{l}\text { Core, section, } \\
\text { interval }(\mathrm{cm})\end{array}$ & $\begin{array}{l}\text { Depth } \\
\text { (mbsf) }\end{array}$ & Taxon & $\begin{array}{l}\text { Size } \\
(\mu \mathrm{m})\end{array}$ & $\delta^{18} \mathrm{O}$ & $\delta^{13} \mathrm{C}$ & $N$ \\
\hline \multicolumn{7}{|c|}{ Planktonic foraminifers } \\
\hline \multicolumn{7}{|c|}{ 171B-1051B- } \\
\hline $2 \mathrm{H}-1,0-2$ & 4.80 & M. spinulosa & $250-355$ & -0.25 & 2.44 & 20 \\
\hline $2 \mathrm{H}-1,10-12$ & 4.90 & M. spinulosa & $250-355$ & -0.71 & 2.25 & 14 \\
\hline $2 \mathrm{H}-1,20-22$ & 5.00 & M. spinulosa & $250-355$ & -0.46 & 2.48 & 20 \\
\hline $2 \mathrm{H}-1,0-32$ & 5.10 & M. spinulosa & $250-355$ & -0.12 & 2.87 & 20 \\
\hline $2 \mathrm{H}-1,50-52$ & 5.30 & M. spinulosa & $250-355$ & -0.44 & 2.79 & 20 \\
\hline $2 \mathrm{H}-1,60-62$ & 5.40 & M. spinulosa & $250-355$ & -1.11 & 1.64 & 20 \\
\hline $2 \mathrm{H}-1,70-72$ & 5.50 & M. spinulosa & $250-355$ & -1.19 & 1.34 & 13 \\
\hline $2 \mathrm{H}-1,80-82$ & 5.60 & M. spinulosa & $250-355$ & -0.33 & 2.68 & 20 \\
\hline $2 \mathrm{H}-1,90-92$ & 5.70 & M. spinulosa & $250-355$ & -0.21 & 2.59 & 15 \\
\hline $2 \mathrm{H}-1,100-102$ & 5.80 & M. crassata & $250-355$ & 0.17 & 3.07 & 17 \\
\hline $2 \mathrm{H}-1,110-112$ & 5.90 & M. spinulosa & $250-355$ & -0.63 & 2.18 & 11 \\
\hline $2 \mathrm{H}-1,120-122$ & 6.00 & M. spinulosa & $250-355$ & -1.05 & 2.15 & 13 \\
\hline $2 \mathrm{H}-1,130-132$ & 6.10 & M. spinulosa & $250-355$ & -0.51 & 2.62 & 14 \\
\hline $2 \mathrm{H}-1,140-142$ & 6.20 & M. crassata & $250-355$ & -0.01 & 2.93 & 20 \\
\hline $2 \mathrm{H}-2,2-4$ & 6.30 & M. spinulosa & $250-355$ & -0.49 & 2.43 & 13 \\
\hline $2 \mathrm{H}-2,10-12$ & 6.40 & M. spinulosa & $250-355$ & -0.43 & 2.40 & 20 \\
\hline $2 \mathrm{H}-2,20-22$ & 6.50 & M. spinulosa & $250-355$ & -0.82 & 1.70 & 5 \\
\hline $2 \mathrm{H}-2,30-32$ & 6.60 & M. spinulosa & $250-355$ & -0.88 & 1.76 & 10 \\
\hline $2 \mathrm{H}-2,40-42$ & 6.70 & M. spinulosa & $250-355$ & -0.11 & 2.43 & 13 \\
\hline $2 \mathrm{H}-2,50-52$ & 6.80 & M. spinulosa & $250-355$ & -0.13 & 2.44 & 13 \\
\hline $2 \mathrm{H}-2,60-62$ & 6.90 & M. spinulosa & $250-355$ & & 1.30 & 18 \\
\hline $2 \mathrm{H}-2,60-62$ & 6.90 & M. crassata & $250-355$ & 0.15 & 2.99 & 20 \\
\hline $2 \mathrm{H}-2,80-82$ & 7.10 & M. spinulosa & $250-355$ & -1.03 & 1.37 & 9 \\
\hline $2 \mathrm{H}-2,80-82$ & 7.10 & M. crassata & $250-355$ & 0.16 & 3.09 & 20 \\
\hline $2 \mathrm{H}-2,90-92$ & 7.20 & M. crassata & $250-355$ & -0.12 & 3.16 & 20 \\
\hline $2 \mathrm{H}-2,100-102$ & 7.30 & M. spinulosa & $250-355$ & -1.53 & 0.74 & 17 \\
\hline $2 \mathrm{H}-2,110-112$ & 7.40 & M. spinulosa & $250-355$ & & 2.78 & 19 \\
\hline $2 \mathrm{H}-2,120-122$ & 7.50 & M. spinulosa & $250-355$ & -0.39 & 2.45 & 15 \\
\hline $2 \mathrm{H}-2,130-132$ & 7.60 & M. spinulosa & $250-355$ & -0.58 & 2.50 & 20 \\
\hline $2 \mathrm{H}-3,0-2$ & 7.80 & M. spinulosa & $250-355$ & -0.44 & 2.80 & 20 \\
\hline $2 \mathrm{H}-3,20-22$ & 8.00 & M. spinulosa & $250-355$ & -0.22 & 2.93 & 20 \\
\hline $2 \mathrm{H}-3,30-32$ & 8.10 & M. spinulosa & $250-355$ & -0.59 & 2.63 & 20 \\
\hline $2 \mathrm{H}-3,40-42$ & 8.20 & M. spinulosa & $250-355$ & -0.28 & 2.88 & 20 \\
\hline $2 \mathrm{H}-3,50-52$ & 8.30 & M. spinulosa & $250-355$ & -0.74 & 2.55 & 13 \\
\hline $2 \mathrm{H}-3,60-62$ & 8.40 & M. spinulosa & $250-355$ & -0.44 & 2.70 & 16 \\
\hline $2 \mathrm{H}-3,70-72$ & 8.50 & M. spinulosa & $250-355$ & -0.83 & 2.50 & 7 \\
\hline $2 \mathrm{H}-3,80-82$ & 8.60 & M. spinulosa & $250-355$ & -0.08 & 2.74 & 14 \\
\hline $2 \mathrm{H}-3,90-92$ & 8.70 & M. spinulosa & $250-355$ & -0.35 & 2.49 & 9 \\
\hline $2 \mathrm{H}-3,100-102$ & 8.80 & M. spinulosa & $250-355$ & -0.85 & 2.05 & 6 \\
\hline $2 \mathrm{H}-3,110-112$ & 8.90 & M. crassata & $250-355$ & -0.20 & 2.88 & 12 \\
\hline $2 \mathrm{H}-3,120-122$ & 9.00 & M. crassata & $250-355$ & -0.15 & 2.94 & 17 \\
\hline $2 \mathrm{H}-3,130-132$ & 9.10 & M. crassata & $250-355$ & 0.00 & 3.02 & 10 \\
\hline $2 \mathrm{H}-3,130-132$ & 9.10 & M. crassata & $250-355$ & -0.19 & 2.87 & 10 \\
\hline $2 \mathrm{H}-3,140-142$ & 9.20 & M. crassata & $250-355$ & -0.17 & 2.94 & 12 \\
\hline $2 \mathrm{H}-4,10-12$ & 9.40 & M. spinulosa & $250-355$ & -1.46 & 1.34 & 11 \\
\hline $2 \mathrm{H}-4,20-22$ & 9.50 & M. spinulosa & $250-355$ & -1.43 & 1.24 & 10 \\
\hline $2 \mathrm{H}-4,30-32$ & 9.60 & M. spinulosa & $250-355$ & -1.67 & & 17 \\
\hline $2 \mathrm{H}-4,30-32$ & 9.60 & M. spinulosa & $250-355$ & -1.47 & & 17 \\
\hline $2 \mathrm{H}-4,30-32$ & 9.60 & M. crassata & $250-355$ & 0.19 & 2.93 & 20 \\
\hline $2 \mathrm{H}-4,40-42$ & 9.70 & M. spinulosa & $250-355$ & -1.30 & 1.92 & 14 \\
\hline $2 \mathrm{H}-4,50-52$ & 9.80 & M. spinulosa & $250-355$ & -0.80 & 2.13 & 16 \\
\hline $2 \mathrm{H}-4,60-62$ & 9.90 & M. spinulosa & $250-355$ & -0.80 & 2.27 & 18 \\
\hline $2 \mathrm{H}-4,70-72$ & 10.00 & M. crassata & $250-355$ & -0.10 & 3.09 & 17 \\
\hline $2 \mathrm{H}-4,100-102$ & 10.30 & M. crassata & $250-355$ & -0.28 & 3.16 & 8 \\
\hline $2 \mathrm{H}-4,110-112$ & 10.40 & M. crassata & $250-355$ & 0.00 & 2.79 & 20 \\
\hline $2 \mathrm{H}-4,120-122$ & 10.50 & M. crassata & $250-355$ & -0.13 & 2.84 & 20 \\
\hline $2 \mathrm{H}-4,140-142$ & 10.70 & M. crassata & $250-355$ & -0.15 & 2.81 & 20 \\
\hline $2 \mathrm{H}-5,0-2$ & 10.80 & M. spinulosa & $250-355$ & & 1.15 & 11 \\
\hline $2 \mathrm{H}-5,0-2$ & 10.80 & M. crassata & $250-355$ & -0.12 & 2.87 & 19 \\
\hline $2 \mathrm{H}-5,10-12$ & 10.90 & M. spinulosa & $250-355$ & -0.67 & 2.26 & 11 \\
\hline $2 \mathrm{H}-5,20-22$ & 11.00 & M. spinulosa & $250-355$ & -0.64 & 2.88 & 17 \\
\hline $2 \mathrm{H}-5,30-32$ & 11.10 & M. spinulosa & $250-355$ & & 1.72 & 8 \\
\hline $2 \mathrm{H}-5,40-42$ & 11.20 & M. spinulosa & $250-355$ & -0.49 & 3.01 & 15 \\
\hline $2 \mathrm{H}-5,50-52$ & 11.30 & M. spinulosa & $250-355$ & -0.95 & 1.79 & 12 \\
\hline $2 \mathrm{H}-5,60-62$ & 11.40 & M. spinulosa & $250-355$ & -0.18 & 2.77 & 20 \\
\hline $2 \mathrm{H}-5,70-72$ & 11.50 & M. spinulosa & $250-355$ & -0.14 & 2.83 & 19 \\
\hline
\end{tabular}


B.S. WADE ET AL.

DATA Report: Isotope Stratigraphy, LATE MidDle EOCENE

Table T1 (continued).

\begin{tabular}{|c|c|c|c|c|c|c|}
\hline $\begin{array}{l}\text { Core, section, } \\
\text { interval }(\mathrm{cm})\end{array}$ & $\begin{array}{l}\text { Depth } \\
\text { (mbsf) }\end{array}$ & Taxon & $\begin{array}{l}\text { Size } \\
(\mu \mathrm{m})\end{array}$ & $\delta^{18} \mathrm{O}$ & $\delta^{13} \mathrm{C}$ & $N$ \\
\hline $2 \mathrm{H}-5,90-92$ & 11.70 & M. spinulosa & $250-355$ & -0.32 & 2.51 & 14 \\
\hline $2 \mathrm{H}-5,100-102$ & 11.80 & M. spinulosa & $250-355$ & -0.78 & 2.44 & 19 \\
\hline $2 \mathrm{H}-5,110-112$ & 11.90 & M. spinulosa & $250-355$ & -0.47 & 2.51 & 9 \\
\hline $2 \mathrm{H}-5,120-122$ & 12.00 & M. spinulosa & $250-355$ & -0.19 & 2.86 & 20 \\
\hline $2 \mathrm{H}-5,130-132$ & 12.10 & M. spinulosa & $250-355$ & -0.52 & 2.29 & 13 \\
\hline $2 \mathrm{H}-5,140-142$ & 12.20 & M. spinulosa & $250-355$ & -0.43 & 2.41 & 11 \\
\hline $2 \mathrm{H}-6,0-3$ & 12.40 & M. crassata & $250-355$ & 0.20 & 2.83 & 18 \\
\hline $2 \mathrm{H}-6,10-13$ & 12.40 & M. spinulosa & $250-355$ & -0.24 & 2.68 & 17 \\
\hline $2 \mathrm{H}-6,20-23$ & 12.50 & M. spinulosa & $250-355$ & -0.14 & 3.08 & 20 \\
\hline $2 \mathrm{H}-6,30-33$ & 12.60 & M. spinulosa & $250-355$ & -0.93 & 2.14 & 20 \\
\hline $2 \mathrm{H}-6,40-43$ & 12.70 & M. crassata & $250-355$ & -0.21 & 2.69 & 20 \\
\hline $2 \mathrm{H}-6,50-53$ & 12.80 & M. crassata & $250-355$ & -0.17 & 2.68 & 20 \\
\hline $2 \mathrm{H}-6,70-73$ & 13.00 & M. spinulosa & $250-355$ & -0.93 & 2.42 & 18 \\
\hline $2 \mathrm{H}-6,80-83$ & 13.10 & M. spinulosa & $250-355$ & & 2.62 & 17 \\
\hline $2 \mathrm{H}-6,90-93$ & 13.20 & M. crassata & $250-355$ & -0.30 & 2.59 & 20 \\
\hline $2 \mathrm{H}-6,100-103$ & 13.30 & M. spinulosa & $250-355$ & -0.87 & 2.39 & 19 \\
\hline $2 \mathrm{H}-6,110-113$ & 13.40 & M. spinulosa & $250-355$ & -0.47 & 2.22 & 16 \\
\hline $2 \mathrm{H}-6,120-123$ & 13.50 & M. spinulosa & $250-355$ & -0.87 & 2.26 & 13 \\
\hline $2 \mathrm{H}-6,130-133$ & 13.60 & M. spinulosa & $250-355$ & -0.91 & 1.96 & 18 \\
\hline $2 \mathrm{H}-6,140-143$ & 13.70 & M. spinulosa & $250-355$ & -0.72 & 2.11 & 20 \\
\hline $2 \mathrm{H}-7,0-3$ & 13.80 & M. spinulosa & $250-355$ & -0.62 & 2.41 & 20 \\
\hline $2 \mathrm{H}-7,10-13$ & 13.90 & M. crassata & $250-355$ & -0.17 & 2.85 & 20 \\
\hline $2 \mathrm{H}-7,20-23$ & 14.00 & M. spinulosa & $250-355$ & -0.82 & 1.97 & 11 \\
\hline $2 \mathrm{H}-7,30-33$ & 14.10 & M. crassata & $250-355$ & -0.18 & 2.75 & 11 \\
\hline $2 \mathrm{H}-7,40-43$ & 14.20 & M. spinulosa & $250-355$ & -0.44 & 2.73 & 18 \\
\hline $2 \mathrm{H}-7,70-73$ & 14.30 & M. spinulosa & $250-355$ & -0.58 & 2.45 & 20 \\
\hline $3 \mathrm{H}-1,10-13$ & 14.40 & M. spinulosa & $250-355$ & -0.35 & 2.64 & 20 \\
\hline $3 \mathrm{H}-1,10-13$ & 14.40 & M. crassata & $250-355$ & -0.27 & 2.50 & 21 \\
\hline $3 \mathrm{H}-1,20-23$ & 14.50 & M. spinulosa & $250-355$ & -0.25 & 2.79 & 16 \\
\hline $3 \mathrm{H}-1,30-33$ & 14.60 & M. spinulosa & $250-355$ & -0.10 & 2.77 & 20 \\
\hline $3 \mathrm{H}-1,30-33$ & 14.60 & M. crassata & $250-355$ & -0.06 & 2.63 & 20 \\
\hline $3 \mathrm{H}-1,40-43$ & 14.70 & M. spinulosa & $250-355$ & -0.14 & 2.77 & 12 \\
\hline $3 \mathrm{H}-1,50-53$ & 14.80 & M. spinulosa & $250-355$ & -0.18 & 2.67 & 20 \\
\hline $3 \mathrm{H}-1,60-63$ & 14.90 & M. spinulosa & $250-355$ & -0.03 & 2.54 & 20 \\
\hline $3 \mathrm{H}-1,70-73$ & 15.00 & M. spinulosa & $250-355$ & -0.20 & 2.73 & 19 \\
\hline $3 \mathrm{H}-1,80-83$ & 15.10 & M. spinulosa & $250-355$ & -0.57 & 2.69 & 20 \\
\hline $3 \mathrm{H}-1,90-93$ & 15.20 & M. spinulosa & $250-355$ & -0.47 & 2.49 & 20 \\
\hline $3 \mathrm{H}-1,98-100$ & 15.27 & M. spinulosa & $250-355$ & -0.40 & 2.79 & 20 \\
\hline $3 \mathrm{H}-2,10-13$ & 15.90 & M. spinulosa & $250-355$ & -0.11 & 2.76 & 20 \\
\hline $3 \mathrm{H}-2,20-23$ & 16.00 & M. spinulosa & $250-355$ & -0.66 & 2.03 & 17 \\
\hline $3 \mathrm{H}-2,30-33$ & 16.10 & M. spinulosa & $250-355$ & -0.89 & 2.33 & 20 \\
\hline $3 \mathrm{H}-2,40-43$ & 16.20 & M. spinulosa & $250-355$ & -0.55 & 2.21 & 20 \\
\hline $3 \mathrm{H}-2,50-53$ & 16.30 & M. spinulosa & $250-355$ & -0.88 & 2.28 & 15 \\
\hline $3 \mathrm{H}-2,60-63$ & 16.40 & M. spinulosa & $250-355$ & -0.64 & 2.52 & 20 \\
\hline $3 \mathrm{H}-2,70-73$ & 16.50 & M. spinulosa & $250-355$ & -0.65 & 2.37 & 20 \\
\hline $3 \mathrm{H}-2,80-83$ & 16.60 & M. spinulosa & $250-355$ & -0.54 & 2.42 & 20 \\
\hline $3 \mathrm{H}-2,90-93$ & 16.70 & M. spinulosa & $250-355$ & -0.52 & 2.60 & 20 \\
\hline $3 \mathrm{H}-2,100-103$ & 16.80 & M. spinulosa & $250-355$ & -0.39 & 2.40 & 20 \\
\hline $3 \mathrm{H}-2,110-113$ & 16.90 & M. spinulosa & $250-355$ & -0.45 & 2.52 & 19 \\
\hline $3 \mathrm{H}-2,120-123$ & 17.00 & M. spinulosa & $250-355$ & -0.30 & 2.49 & 17 \\
\hline $3 \mathrm{H}-2,125-127$ & 17.05 & M. spinulosa & $250-355$ & -0.23 & 2.83 & 20 \\
\hline $3 \mathrm{H}-2,145-147$ & 17.25 & M. spinulosa & $250-355$ & -0.51 & 2.76 & 19 \\
\hline $3 \mathrm{H}-3,0-3$ & 17.30 & M. spinulosa & $250-355$ & -0.50 & 2.57 & 20 \\
\hline $3 \mathrm{H}-3,10-13$ & 17.40 & M. spinulosa & $250-355$ & -0.71 & 2.40 & 10 \\
\hline $3 \mathrm{H}-3,10-13$ & 17.40 & M. crassata & $250-355$ & -0.36 & 2.70 & 14 \\
\hline $3 \mathrm{H}-3,20-23$ & 17.50 & M. spinulosa & $250-355$ & -0.62 & 2.42 & 11 \\
\hline $3 \mathrm{H}-3,20-23$ & 17.50 & A. praetopilensis & $250-355$ & -0.29 & 2.79 & 15 \\
\hline $3 \mathrm{H}-3,30-33$ & 17.60 & M. spinulosa & $250-355$ & -0.29 & 2.59 & 14 \\
\hline $3 \mathrm{H}-3,40-43$ & 17.70 & M. spinulosa & $250-355$ & -0.28 & 2.79 & 16 \\
\hline $3 \mathrm{H}-3,50-53$ & 17.80 & M. spinulosa & $250-355$ & -0.85 & 2.20 & 8 \\
\hline $3 \mathrm{H}-3,50-53$ & 17.80 & A. praetopilensis & $250-355$ & -0.03 & 2.63 & 14 \\
\hline $3 \mathrm{H}-3,50-53$ & 17.80 & G. mexicana & $250-355$ & -0.27 & 1.99 & 12 \\
\hline $3 \mathrm{H}-3,60-63$ & 17.90 & M. spinulosa & $250-355$ & -0.37 & 2.77 & 16 \\
\hline $3 \mathrm{H}-3,70-73$ & 18.00 & M. spinulosa & $250-355$ & -0.60 & 2.61 & 15 \\
\hline $3 \mathrm{H}-3,70-73$ & 18.00 & A. praetopilensis & $250-355$ & -0.17 & 2.74 & 19 \\
\hline $3 \mathrm{H}-3,70-73$ & 18.00 & M. crassata & $250-355$ & -0.69 & 2.59 & 13 \\
\hline $3 \mathrm{H}-3,80-83$ & 18.10 & M. spinulosa & $250-355$ & -0.57 & 2.63 & 13 \\
\hline $3 \mathrm{H}-3,90-93$ & 18.20 & M. spinulosa & $250-355$ & -0.69 & 2.51 & 11 \\
\hline $3 \mathrm{H}-3,90-93$ & 18.20 & A. praetopilensis & $250-355$ & 0.22 & 2.99 & 21 \\
\hline
\end{tabular}


B.S. WADE ET AL.

DATA Report: Isotope Stratigraphy, LATE MidDle EOCENE

Table T1 (continued).

\begin{tabular}{|c|c|c|c|c|c|c|}
\hline $\begin{array}{l}\text { Core, section, } \\
\text { interval }(\mathrm{cm})\end{array}$ & $\begin{array}{l}\text { Depth } \\
\text { (mbsf) }\end{array}$ & Taxon & $\begin{array}{l}\text { Size } \\
(\mu \mathrm{m})\end{array}$ & $\delta^{18} \mathrm{O}$ & $\delta^{13} \mathrm{C}$ & $N$ \\
\hline $3 \mathrm{H}-3,100-103$ & 18.30 & M. spinulosa & $250-355$ & -0.76 & 2.29 & 10 \\
\hline $3 \mathrm{H}-3,100-103$ & 18.30 & A. praetopilensis & $250-355$ & -0.23 & 2.79 & 20 \\
\hline $3 \mathrm{H}-3,110-113$ & 18.40 & M. spinulosa & $250-355$ & -0.61 & 2.67 & 12 \\
\hline $3 \mathrm{H}-3,120-123$ & 18.50 & M. spinulosa & $250-355$ & -0.63 & 2.43 & 8 \\
\hline $3 \mathrm{H}-3,120-123$ & 18.50 & A. praetopilensis & $250-355$ & -0.71 & 2.25 & 21 \\
\hline $3 \mathrm{H}-3,130-133$ & 18.60 & M. spinulosa & $250-355$ & -0.76 & 2.52 & 14 \\
\hline $3 \mathrm{H}-3,140-143$ & 18.70 & A. praetopilensis & $250-355$ & -0.21 & 2.63 & 21 \\
\hline $3 \mathrm{H}-4,0-3$ & 18.80 & G. mexicana & $250-355$ & -0.24 & 1.74 & 20 \\
\hline $3 \mathrm{H}-4,10-13$ & 18.90 & M. spinulosa & $250-355$ & -1.08 & 1.93 & 14 \\
\hline $3 \mathrm{H}-4,20-23$ & 19.00 & M. spinulosa & $250-355$ & -1.35 & 2.04 & 11 \\
\hline $3 \mathrm{H}-4,30-33$ & 19.10 & G. mexicana & $250-355$ & -0.47 & 1.99 & 20 \\
\hline $3 \mathrm{H}-4,40-43$ & 19.20 & M. spinulosa & $250-355$ & -1.31 & 2.11 & 20 \\
\hline $3 \mathrm{H}-4,50-53$ & 19.30 & M. spinulosa & $250-355$ & -1.07 & 2.18 & 20 \\
\hline $3 \mathrm{H}-4,60-63$ & 19.40 & M. spinulosa & $250-355$ & -1.21 & 2.10 & 20 \\
\hline $3 \mathrm{H}-4,70-73$ & 19.50 & M. spinulosa & $250-355$ & -0.94 & 2.51 & 16 \\
\hline $3 \mathrm{H}-4,80-83$ & 19.60 & M. spinulosa & $250-355$ & -1.31 & 2.09 & 12 \\
\hline $3 \mathrm{H}-4,90-93$ & 19.70 & M. spinulosa & $250-355$ & -0.98 & 2.14 & 20 \\
\hline $3 \mathrm{H}-4,100-103$ & 19.80 & M. spinulosa & $250-355$ & -0.81 & 2.30 & 20 \\
\hline $3 \mathrm{H}-4,110-113$ & 19.90 & M. spinulosa & $250-355$ & -1.03 & 2.04 & 14 \\
\hline $3 \mathrm{H}-4,120-123$ & 20.00 & M. spinulosa & $250-355$ & -0.75 & 2.25 & 20 \\
\hline $3 \mathrm{H}-4,120-123$ & 20.00 & M. spinulosa & $250-355$ & -0.77 & 2.42 & 20 \\
\hline $3 \mathrm{H}-4,130-133$ & 20.10 & M. spinulosa & $250-355$ & -0.68 & 2.38 & 12 \\
\hline $3 \mathrm{H}-4,140-143$ & 20.20 & M. spinulosa & $250-355$ & -1.01 & 2.35 & 20 \\
\hline $3 \mathrm{H}-4,140-143$ & 20.20 & M. spinulosa & $250-355$ & -1.21 & & 5 \\
\hline $3 \mathrm{H}-5,0-3$ & 20.30 & M. spinulosa & $250-355$ & -0.58 & 2.58 & 20 \\
\hline $3 \mathrm{H}-5,0-3$ & 20.30 & M. spinulosa & $250-355$ & -0.47 & 2.71 & 20 \\
\hline $3 \mathrm{H}-5,10-13$ & 20.40 & M. spinulosa & $250-355$ & -0.80 & 2.80 & 20 \\
\hline $3 \mathrm{H}-5,10-13$ & 20.40 & M. spinulosa & $250-355$ & -0.47 & 2.71 & 20 \\
\hline $3 \mathrm{H}-5,20-23$ & 20.50 & M. spinulosa & $250-355$ & -0.63 & 2.69 & 20 \\
\hline $3 \mathrm{H}-5,30-33$ & 20.60 & M. spinulosa & $250-355$ & -0.63 & 2.88 & 20 \\
\hline $3 \mathrm{H}-5,40-43$ & 20.70 & M. spinulosa & $250-355$ & -0.65 & 2.80 & 20 \\
\hline $3 \mathrm{H}-5,50-53$ & 20.80 & M. spinulosa & $250-355$ & -0.74 & 2.90 & 20 \\
\hline $3 \mathrm{H}-5,50-53$ & 20.80 & M. spinulosa & $125-250$ & -0.50 & 2.24 & 18 \\
\hline $3 \mathrm{H}-5,60-63$ & 20.90 & M. spinulosa & $250-355$ & -0.51 & 2.81 & 20 \\
\hline $3 \mathrm{H}-5,60-63$ & 20.90 & M. spinulosa & $125-250$ & -0.53 & 2.33 & 23 \\
\hline $3 \mathrm{H}-5,70-73$ & 21.00 & M. spinulosa & $250-355$ & -0.67 & 2.63 & 20 \\
\hline $3 \mathrm{H}-5,70-73$ & 21.00 & M. spinulosa & $250-355$ & & 2.89 & 6 \\
\hline $3 \mathrm{H}-5,80-83$ & 21.10 & M. spinulosa & $250-355$ & -0.77 & 2.87 & 20 \\
\hline $3 \mathrm{H}-5,90-93$ & 21.20 & M. spinulosa & $250-355$ & -0.46 & 2.87 & 20 \\
\hline $3 \mathrm{H}-5,100-103$ & 21.30 & M. spinulosa & $250-355$ & -0.65 & 2.74 & 20 \\
\hline $3 \mathrm{H}-5,110-113$ & 21.40 & M. spinulosa & $250-355$ & -0.81 & 2.73 & 20 \\
\hline $3 \mathrm{H}-5,120-123$ & 21.50 & M. spinulosa & $250-355$ & -1.01 & 2.65 & 20 \\
\hline $3 \mathrm{H}-5,120-123$ & 21.50 & M. spinulosa & $250-355$ & -0.76 & 2.45 & 20 \\
\hline $3 \mathrm{H}-5,130-133$ & 21.60 & M. spinulosa & $250-355$ & -0.66 & 2.74 & 20 \\
\hline $3 \mathrm{H}-5,140-143$ & 21.70 & M. spinulosa & $250-355$ & -0.36 & 2.79 & 20 \\
\hline $3 \mathrm{H}-6,0-2$ & 21.80 & M. spinulosa & $250-355$ & -0.47 & 2.94 & 20 \\
\hline $3 \mathrm{H}-6,10-12$ & 21.90 & M. spinulosa & $250-355$ & -0.39 & 2.89 & 20 \\
\hline $3 \mathrm{H}-6,20-22$ & 22.00 & M. spinulosa & $250-355$ & -0.98 & 2.83 & 21 \\
\hline $3 \mathrm{H}-6,30-32$ & 22.10 & M. spinulosa & $250-355$ & -0.28 & 2.98 & 21 \\
\hline $3 \mathrm{H}-6,40-42$ & 22.20 & M. spinulosa & $250-355$ & -0.40 & 3.26 & 20 \\
\hline $3 \mathrm{H}-6,50-52$ & 22.30 & M. spinulosa & $250-355$ & -0.21 & 3.15 & 20 \\
\hline $3 \mathrm{H}-6,60-62$ & 22.40 & M. spinulosa & $250-355$ & -0.54 & 3.32 & 19 \\
\hline $3 \mathrm{H}-6,70-72$ & 22.50 & M. spinulosa & $250-355$ & -0.45 & 3.08 & 20 \\
\hline $3 \mathrm{H}-6,80-82$ & 22.60 & M. spinulosa & $250-355$ & -0.30 & 3.06 & 12 \\
\hline $3 \mathrm{H}-6,90-92$ & 22.70 & M. spinulosa & $250-355$ & -0.92 & 3.24 & 5 \\
\hline $3 \mathrm{H}-6,100-102$ & 22.80 & M. spinulosa & $250-355$ & -0.60 & 2.83 & 5 \\
\hline $3 \mathrm{H}-6,110-112$ & 22.90 & Morozovella spp. & $250-355$ & -0.63 & 2.92 & 7 \\
\hline $3 \mathrm{H}-6,110-112$ & 22.90 & A. praetopilensis & $250-355$ & -0.29 & 2.64 & 13 \\
\hline $3 \mathrm{H}-6,120-122$ & 23.00 & M. spinulosa & $250-355$ & -1.05 & 3.13 & 5 \\
\hline $3 \mathrm{H}-6,130-132$ & 23.10 & Morozovella spp. & $250-355$ & -0.57 & 2.94 & 11 \\
\hline $4 \mathrm{H}-1,60-62$ & 24.40 & M. spinulosa & $250-355$ & -0.48 & 3.34 & 18 \\
\hline $4 \mathrm{H}-1,70-72$ & 24.50 & M. spinulosa & $250-355$ & -0.42 & 3.09 & 20 \\
\hline $4 \mathrm{H}-1,80-82$ & 24.60 & M. spinulosa & $250-355$ & -0.61 & 3.41 & 9 \\
\hline $4 \mathrm{H}-1,90-92$ & 24.70 & M. spinulosa & $250-355$ & -0.48 & 2.98 & 15 \\
\hline $4 \mathrm{H}-1,100-102$ & 24.80 & M. spinulosa & $250-355$ & -0.47 & 3.15 & 16 \\
\hline $4 \mathrm{H}-1,110-112$ & 24.90 & M. spinulosa & $250-355$ & -0.63 & 2.95 & 17 \\
\hline $4 \mathrm{H}-1,120-122$ & 25.00 & M. spinulosa & $250-355$ & -0.53 & 3.15 & 13 \\
\hline $4 \mathrm{H}-1,130-132$ & 25.10 & M. spinulosa & $250-355$ & -0.48 & 3.03 & 19 \\
\hline $4 \mathrm{H}-1,140-142$ & 25.20 & M. spinulosa & $250-355$ & -0.56 & 3.10 & 20 \\
\hline
\end{tabular}


B.S. WADE ET AL.

DATA Report: Isotope Stratigraphy, LATE MIDDLE EOCENE

Table T1 (continued).

\begin{tabular}{|c|c|c|c|c|c|c|}
\hline $\begin{array}{c}\text { Core, section, } \\
\text { interval }(\mathrm{cm})\end{array}$ & $\begin{array}{l}\text { Depth } \\
\text { (mbsf) }\end{array}$ & Taxon & $\begin{array}{l}\text { Size } \\
(\mu \mathrm{m})\end{array}$ & $\delta^{18} \mathrm{O}$ & $\delta^{13} \mathrm{C}$ & $N$ \\
\hline $4 \mathrm{H}-2,10-12$ & 25.40 & M. spinulosa & $250-355$ & -0.69 & 3.26 & 17 \\
\hline $4 \mathrm{H}-2,20-22$ & 25.50 & M. spinulosa & $250-355$ & -0.55 & 3.17 & 20 \\
\hline $4 \mathrm{H}-2,30-32$ & 25.60 & M. spinulosa & $250-355$ & -0.68 & 3.18 & 21 \\
\hline $4 \mathrm{H}-2,40-42$ & 25.70 & M. spinulosa & $250-355$ & -0.60 & 3.15 & 20 \\
\hline $4 \mathrm{H}-2,50-52$ & 25.80 & M. spinulosa & $250-355$ & -0.55 & 3.03 & 20 \\
\hline $4 \mathrm{H}-2,60-62$ & 25.90 & M. spinulosa & $250-355$ & -0.68 & 3.18 & 9 \\
\hline $4 \mathrm{H}-2,70-72$ & 26.00 & M. spinulosa & $250-355$ & -0.55 & 3.24 & 16 \\
\hline $4 \mathrm{H}-2,80-82$ & 26.10 & M. spinulosa & $250-355$ & -0.59 & 2.96 & 21 \\
\hline $4 \mathrm{H}-2,90-92$ & 26.20 & M. spinulosa & $250-355$ & -0.41 & 3.16 & 15 \\
\hline $4 \mathrm{H}-2,100-102$ & 26.30 & M. spinulosa & $250-355$ & -0.72 & 3.29 & 6 \\
\hline $4 \mathrm{H}-2,110-112$ & 26.40 & M. crassata & $250-355$ & -0.49 & 3.03 & 17 \\
\hline $4 \mathrm{H}-2,120-122$ & 26.50 & M. spinulosa & $250-355$ & -0.52 & 3.27 & 10 \\
\hline $4 \mathrm{H}-2,120-122$ & 26.50 & M. spinulosa & $250-355$ & -0.52 & 3.12 & 12 \\
\hline $4 \mathrm{H}-2,130-132$ & 26.60 & M. spinulosa & $250-355$ & -0.54 & 3.31 & 20 \\
\hline $4 \mathrm{H}-2,140-142$ & 26.70 & M. spinulosa & $250-355$ & -0.59 & 3.20 & 18 \\
\hline $4 \mathrm{H}-3,0-2$ & 26.80 & M. spinulosa & $250-355$ & -0.68 & 3.09 & 21 \\
\hline $4 \mathrm{H}-3,10-12$ & 26.90 & M. spinulosa & $250-355$ & -0.70 & 3.17 & 20 \\
\hline $4 \mathrm{H}-3,20-22$ & 27.00 & M. spinulosa & $250-355$ & -0.66 & 3.16 & 20 \\
\hline $4 \mathrm{H}-3,30-32$ & 27.10 & M. spinulosa & $250-355$ & -0.78 & 3.11 & 21 \\
\hline $4 \mathrm{H}-3,40-42$ & 27.20 & M. spinulosa & $250-355$ & -0.53 & 3.11 & 20 \\
\hline $4 \mathrm{H}-3,50-52$ & 27.30 & M. spinulosa & $250-355$ & -0.86 & 3.01 & 20 \\
\hline $4 \mathrm{H}-3,60-62$ & 27.40 & M. spinulosa & $250-355$ & -0.86 & 3.10 & 21 \\
\hline $4 \mathrm{H}-3,70-72$ & 27.50 & M. spinulosa & $250-355$ & -0.61 & 3.19 & 20 \\
\hline $4 \mathrm{H}-3,80-82$ & 27.60 & M. spinulosa & $250-355$ & -0.67 & 3.07 & 13 \\
\hline \multicolumn{7}{|c|}{ Benthic foraminifers } \\
\hline $2 \mathrm{H}-1,0-2$ & 4.80 & N. truempyi & $<150$ & 1.00 & 1.06 & \\
\hline $2 \mathrm{H}-1,10-12$ & 4.90 & N. truempyi & $<150$ & 1.01 & 1.18 & \\
\hline $2 \mathrm{H}-1,20-22$ & 5.00 & N. truempyi & $<150$ & 1.03 & 1.23 & \\
\hline $2 \mathrm{H}-1,30-32$ & 5.10 & N. truempyi & $<150$ & 0.93 & 1.14 & \\
\hline $2 \mathrm{H}-1,40-42$ & 5.20 & N. truempyi & $<150$ & 1.03 & 1.17 & \\
\hline $2 \mathrm{H}-1,50-52$ & 5.30 & N. truempyi & $<150$ & 0.90 & 1.05 & \\
\hline $2 \mathrm{H}-1,60-62$ & 5.40 & N. truempyi & $<150$ & 0.86 & 1.07 & \\
\hline $2 \mathrm{H}-1,70-72$ & 5.50 & N. truempyi & $<150$ & 0.92 & 1.05 & \\
\hline $2 \mathrm{H}-1,80-82$ & 5.60 & N. truempyi & $<150$ & 0.88 & 1.12 & \\
\hline $2 \mathrm{H}-1,90-92$ & 5.70 & N. truempyi & $<150$ & 0.87 & 1.05 & \\
\hline $2 \mathrm{H}-1,100-102$ & 5.80 & N. truempyi & $<150$ & 0.95 & 1.42 & \\
\hline $2 \mathrm{H}-1,110-112$ & 5.90 & N. truempyi & $<150$ & 0.94 & 1.12 & \\
\hline $2 \mathrm{H}-1,120-122$ & 6.00 & N. truempyi & $<150$ & 0.91 & 1.14 & \\
\hline $2 \mathrm{H}-1,130-132$ & 6.10 & N. truempyi & $<150$ & 0.93 & 1.08 & \\
\hline $2 \mathrm{H}-1,140-142$ & 6.20 & N. truempyi & $<150$ & 0.91 & 1.07 & \\
\hline $2 \mathrm{H}-2,2-4$ & 6.30 & N. truempyi & $<150$ & 0.88 & 1.04 & \\
\hline $2 \mathrm{H}-2,10-12$ & 6.40 & N. truempyi & $<150$ & 0.92 & 1.14 & \\
\hline $2 \mathrm{H}-2,20-22$ & 6.50 & N. truempyi & $<150$ & 0.93 & 1.08 & \\
\hline $2 \mathrm{H}-2,30-32$ & 6.60 & N. truempyi & $<150$ & 0.98 & 1.11 & \\
\hline $2 \mathrm{H}-2,40-42$ & 6.70 & N. truempyi & $<150$ & 0.92 & 1.13 & \\
\hline $2 \mathrm{H}-2,50-52$ & 6.80 & N. truempyi & $<150$ & 0.82 & 1.14 & \\
\hline $2 \mathrm{H}-2,60-62$ & 6.90 & N. truempyi & $<150$ & 0.99 & 1.18 & \\
\hline $2 \mathrm{H}-2,70-72$ & 7.00 & N. truempyi & $<150$ & 0.84 & 1.08 & \\
\hline $2 \mathrm{H}-2,80-82$ & 7.10 & N. truempyi & $<150$ & 0.98 & 1.17 & \\
\hline $2 \mathrm{H}-2,90-92$ & 7.20 & N. truempyi & $<150$ & 0.94 & 1.15 & \\
\hline $2 \mathrm{H}-2,100-102$ & 7.30 & N. truempyi & $<150$ & 0.98 & 1.12 & \\
\hline $2 \mathrm{H}-2,110-112$ & 7.40 & N. truempyi & $<150$ & 0.94 & 1.13 & \\
\hline $2 \mathrm{H}-2,120-122$ & 7.50 & N. truempyi & $<150$ & 0.93 & 1.16 & \\
\hline $2 \mathrm{H}-2,130-132$ & 7.60 & N. truempyi & $<150$ & 0.97 & 1.14 & \\
\hline $2 \mathrm{H}-2,140-142$ & 7.70 & N. truempyi & $<150$ & 0.98 & 1.62 & \\
\hline $2 \mathrm{H}-3,0-2$ & 7.80 & N. truempyi & $<150$ & 0.83 & 0.92 & \\
\hline $2 \mathrm{H}-3,10-12$ & 7.90 & N. truempyi & $<150$ & 0.86 & 0.89 & \\
\hline $2 \mathrm{H}-3,20-22$ & 8.00 & N. truempyi & $<150$ & 0.88 & 1.04 & \\
\hline $2 \mathrm{H}-3,30-32$ & 8.10 & N. truempyi & $<150$ & 0.79 & 0.89 & \\
\hline $2 \mathrm{H}-3,40-42$ & 8.20 & N. truempyi & $<150$ & 0.78 & 0.88 & \\
\hline $2 \mathrm{H}-3,50-52$ & 8.30 & N. truempyi & $<150$ & 0.78 & 0.89 & \\
\hline $2 \mathrm{H}-3,60-62$ & 8.40 & N. truempyi & $<150$ & 0.85 & 0.87 & \\
\hline $2 \mathrm{H}-3,70-72$ & 8.50 & N. truempyi & $<150$ & 0.97 & 0.81 & \\
\hline $2 \mathrm{H}-3,80-82$ & 8.60 & N. truempyi & $<150$ & 0.96 & 0.96 & \\
\hline $2 \mathrm{H}-3,90-92$ & 8.70 & N. truempyi & $<150$ & 0.92 & 0.99 & \\
\hline $2 \mathrm{H}-3,110-112$ & 8.90 & N. truempyi & $<150$ & 0.68 & 0.82 & \\
\hline $2 \mathrm{H}-3,120-122$ & 9.00 & N. truempyi & $<150$ & 0.56 & 0.82 & \\
\hline $2 \mathrm{H}-3,130-132$ & 9.10 & N. truempyi & $<150$ & 0.69 & 0.86 & \\
\hline
\end{tabular}


B.S. WADE ET AL.

DATA Report: Isotope Stratigraphy, LATE MidDle EOCENE

Table T1 (continued).

\begin{tabular}{|c|c|c|c|c|c|c|}
\hline $\begin{array}{l}\text { Core, section, } \\
\text { interval }(\mathrm{cm})\end{array}$ & $\begin{array}{l}\text { Depth } \\
\text { (mbsf) }\end{array}$ & Taxon & $\begin{array}{l}\text { Size } \\
(\mu \mathrm{m})\end{array}$ & $\delta^{18} \mathrm{O}$ & $\delta^{13} \mathrm{C}$ & $N$ \\
\hline $2 \mathrm{H}-3,140-142$ & 9.20 & N. truempyi & $<150$ & 0.57 & 0.84 & \\
\hline $2 \mathrm{H}-4,0-2$ & 9.30 & N. truempyi & $<150$ & 0.76 & 0.89 & \\
\hline $2 \mathrm{H}-4,10-12$ & 9.40 & N. truempyi & $<150$ & 0.67 & 0.77 & \\
\hline $2 \mathrm{H}-4,20-22$ & 9.50 & N. truempyi & $<150$ & 0.78 & 0.91 & \\
\hline $2 \mathrm{H}-4,30-32$ & 9.60 & N. truempyi & $<150$ & 1.00 & 1.06 & \\
\hline $2 \mathrm{H}-4,30-32$ & 9.60 & N. truempyi & $<150$ & 1.00 & 1.06 & \\
\hline $2 \mathrm{H}-4,40-42$ & 9.70 & N. truempyi & $<150$ & 0.71 & 0.97 & \\
\hline $2 \mathrm{H}-4,50-52$ & 9.80 & N. truempyi & $<150$ & 0.78 & 0.95 & \\
\hline $2 \mathrm{H}-4,60-62$ & 9.90 & N. truempyi & $<150$ & 0.72 & 0.91 & \\
\hline $2 \mathrm{H}-4,70-72$ & 10.00 & N. truempyi & $<150$ & 0.75 & 0.88 & \\
\hline $2 \mathrm{H}-4,80-82$ & 10.10 & N. truempyi & $<150$ & 0.63 & 0.93 & \\
\hline $2 \mathrm{H}-4,90-92$ & 10.20 & N. truempyi & $<150$ & 0.75 & 0.85 & \\
\hline $2 \mathrm{H}-4,100-102$ & 10.30 & N. truempyi & $<150$ & 0.62 & 0.77 & \\
\hline $2 \mathrm{H}-4,110-112$ & 10.40 & N. truempyi & $<150$ & 0.69 & 0.95 & \\
\hline $2 \mathrm{H}-4,120-122$ & 10.50 & N. truempyi & $<150$ & 0.69 & 0.85 & \\
\hline $2 \mathrm{H}-4,130-132$ & 10.60 & N. truempyi & $<150$ & 0.61 & 0.86 & \\
\hline $2 \mathrm{H}-4,140-142$ & 10.70 & N. truempyi & $<150$ & 0.75 & 0.84 & \\
\hline $2 \mathrm{H}-5,0-2$ & 10.80 & N. truempyi & $<150$ & 0.69 & 0.81 & \\
\hline $2 \mathrm{H}-5,10-12$ & 10.90 & N. truempyi & $<150$ & 0.67 & 0.96 & \\
\hline $2 \mathrm{H}-5,20-22$ & 11.00 & N. truempyi & $<150$ & 0.74 & 0.83 & \\
\hline $2 \mathrm{H}-5,30-32$ & 11.10 & N. truempyi & $<150$ & 0.82 & 0.87 & \\
\hline $2 \mathrm{H}-5,40-42$ & 11.20 & N. truempyi & $<150$ & 0.84 & 0.96 & \\
\hline $2 \mathrm{H}-5,50-52$ & 11.30 & N. truempyi & $<150$ & 0.85 & 0.86 & \\
\hline $2 \mathrm{H}-5,60-62$ & 11.40 & N. truempyi & $<150$ & 0.70 & 0.83 & \\
\hline $2 \mathrm{H}-5,70-72$ & 11.50 & N. truempyi & $<150$ & 0.79 & 0.88 & \\
\hline $2 \mathrm{H}-5,90-92$ & 11.70 & N. truempyi & $<150$ & 0.85 & 0.62 & \\
\hline $2 \mathrm{H}-5,100-102$ & 11.80 & N. truempyi & $<150$ & 0.84 & 0.72 & \\
\hline $2 \mathrm{H}-5,110-112$ & 11.90 & N. truempyi & $<150$ & 0.85 & 0.90 & \\
\hline $2 \mathrm{H}-5,120-122$ & 12.00 & N. truempyi & $<150$ & 0.79 & 0.84 & \\
\hline $2 \mathrm{H}-5,130-132$ & 12.10 & N. truempyi & $<150$ & 0.69 & 0.91 & \\
\hline $2 \mathrm{H}-5,140-142$ & 12.20 & N. truempyi & $<150$ & 0.75 & 0.88 & \\
\hline $2 \mathrm{H}-6,0-3$ & 12.30 & N. truempyi & $<150$ & 0.75 & 0.84 & \\
\hline $2 \mathrm{H}-6,10-13$ & 12.40 & N. truempyi & $<150$ & 0.72 & 0.75 & \\
\hline $2 \mathrm{H}-6,20-23$ & 12.50 & N. truempyi & $<150$ & 0.56 & 0.62 & \\
\hline $2 \mathrm{H}-6,30-33$ & 12.60 & N. truempyi & $<150$ & 0.61 & 0.77 & \\
\hline $2 \mathrm{H}-6,40-43$ & 12.70 & N. truempyi & $<150$ & 0.94 & 0.88 & \\
\hline $2 \mathrm{H}-6,50-53$ & 12.80 & N. truempyi & $<150$ & 0.87 & 0.83 & \\
\hline $2 \mathrm{H}-6,60-63$ & 12.90 & N. truempyi & $<150$ & 0.83 & 0.87 & \\
\hline $2 \mathrm{H}-6,70-73$ & 13.00 & N. truempyi & $<150$ & 0.74 & 0.71 & \\
\hline $2 \mathrm{H}-6,80-83$ & 13.10 & N. truempyi & $<150$ & 0.71 & 0.39 & \\
\hline $2 \mathrm{H}-6,90-93$ & 13.20 & N. truempyi & $<150$ & 0.60 & 0.60 & \\
\hline $2 \mathrm{H}-6,100-103$ & 13.30 & N. truempyi & $<150$ & 0.58 & 0.63 & \\
\hline $2 \mathrm{H}-6,110-113$ & 13.40 & N. truempyi & $<150$ & 0.80 & 0.76 & \\
\hline $2 \mathrm{H}-6,130-133$ & 13.60 & N. truempyi & $<150$ & 0.85 & 0.87 & \\
\hline $2 \mathrm{H}-6,140-143$ & 13.70 & N. truempyi & $<150$ & 0.72 & 0.89 & \\
\hline $2 \mathrm{H}-7,0-3$ & 13.80 & N. truempyi & $<150$ & 0.84 & 0.79 & \\
\hline $2 \mathrm{H}-7,20-23$ & 14.00 & N. truempyi & $<150$ & 0.73 & 0.69 & \\
\hline $2 \mathrm{H}-7,30-33$ & 14.10 & N. truempyi & $<150$ & 0.64 & 0.69 & \\
\hline $2 \mathrm{H}-7,40-43$ & 14.20 & N. truempyi & $<150$ & 0.71 & 0.74 & \\
\hline $2 \mathrm{H}-7,70-73$ & 14.30 & N. truempyi & $<150$ & 0.59 & 0.63 & \\
\hline $3 \mathrm{H}-1,20-23$ & 14.50 & N. truempyi & $<150$ & 0.63 & 0.32 & \\
\hline $3 \mathrm{H}-1,30-33$ & 14.60 & N. truempyi & $<150$ & 0.76 & 0.83 & \\
\hline $3 \mathrm{H}-1,40-43$ & 14.70 & N. truempyi & $<150$ & 0.69 & 0.70 & \\
\hline $3 \mathrm{H}-1,50-53$ & 14.80 & N. truempyi & $<150$ & 0.48 & & \\
\hline $3 \mathrm{H}-1,60-63$ & 14.90 & N. truempyi & $<150$ & 0.68 & 0.70 & \\
\hline $3 \mathrm{H}-1,70-73$ & 15.00 & N. truempyi & $<150$ & 0.66 & 0.72 & \\
\hline $3 \mathrm{H}-1,80-83$ & 15.10 & N. truempyi & $<150$ & 0.66 & 0.48 & \\
\hline $3 \mathrm{H}-1,90-93$ & 15.20 & N. truempyi & $<150$ & 0.52 & 0.60 & \\
\hline $3 \mathrm{H}-2,20-23$ & 16.00 & N. truempyi & $<150$ & 0.88 & 0.72 & \\
\hline $3 \mathrm{H}-2,30-33$ & 16.10 & N. truempyi & $<150$ & 0.88 & 0.74 & \\
\hline $3 \mathrm{H}-2,40-43$ & 16.20 & N. truempyi & $<150$ & 0.75 & 0.68 & \\
\hline $3 \mathrm{H}-2,50-53$ & 16.30 & N. truempyi & $<150$ & 0.96 & 0.78 & \\
\hline $3 \mathrm{H}-2,60-63$ & 16.40 & N. truempyi & $<150$ & 0.92 & 0.74 & \\
\hline $3 \mathrm{H}-2,70-73$ & 16.50 & N. truempyi & $<150$ & 1.05 & 0.82 & \\
\hline $3 \mathrm{H}-2,80-83$ & 16.60 & N. truempyi & $<150$ & 0.73 & 0.61 & \\
\hline $3 \mathrm{H}-2,90-93$ & 16.70 & N. truempyi & $<150$ & 0.89 & 0.76 & \\
\hline $3 \mathrm{H}-2,100-103$ & 16.80 & N. truempyi & $<150$ & 0.83 & 0.55 & \\
\hline $3 \mathrm{H}-2,110-113$ & 16.90 & N. truempyi & $<150$ & 0.86 & 0.83 & \\
\hline $3 \mathrm{H}-2,120-123$ & 17.00 & N. truempyi & $<150$ & 0.87 & 0.77 & \\
\hline
\end{tabular}


B.S. WADE ET AL.

DATA Report: Isotope Stratigraphy, LATE MidDle EOCENE

Table T1 (continued).

\begin{tabular}{|c|c|c|c|c|c|c|}
\hline $\begin{array}{l}\text { Core, section, } \\
\text { interval }(\mathrm{cm})\end{array}$ & $\begin{array}{l}\text { Depth } \\
\text { (mbsf) }\end{array}$ & Taxon & $\begin{array}{l}\text { Size } \\
(\mu \mathrm{m})\end{array}$ & $\delta^{18} \mathrm{O}$ & $\delta^{13} \mathrm{C}$ & $N$ \\
\hline $3 \mathrm{H}-2,125-127$ & 17.05 & N. truempyi & $<150$ & 0.80 & 0.73 & \\
\hline $3 \mathrm{H}-2,145-147$ & 17.25 & N. truempyi & $<150$ & 0.75 & 0.58 & \\
\hline $3 \mathrm{H}-3,0-3$ & 17.30 & N. truempyi & $<150$ & 0.83 & 0.68 & \\
\hline $3 \mathrm{H}-3,20-23$ & 17.50 & N. truempyi & $<150$ & 0.75 & 0.75 & \\
\hline $3 \mathrm{H}-3,30-33$ & 17.60 & N. truempyi & $<150$ & 0.92 & 0.84 & \\
\hline $3 \mathrm{H}-3,40-43$ & 17.70 & N. truempyi & $<150$ & 0.71 & 0.63 & \\
\hline $3 \mathrm{H}-3,50-53$ & 17.80 & N. truempyi & $<150$ & 0.82 & 0.65 & \\
\hline $3 \mathrm{H}-3,60-63$ & 17.90 & N. truempyi & $<150$ & 0.59 & 0.60 & \\
\hline $3 \mathrm{H}-3,70-73$ & 18.00 & N. truempyi & $<150$ & 0.76 & 0.62 & \\
\hline $3 \mathrm{H}-3,80-83$ & 18.10 & N. truempyi & $<150$ & 0.80 & 0.73 & \\
\hline $3 \mathrm{H}-3,90-93$ & 18.20 & N. truempyi & $<150$ & 0.84 & 0.87 & \\
\hline $3 \mathrm{H}-3,100-103$ & 18.30 & N. truempyi & $<150$ & 0.96 & 0.96 & \\
\hline $3 \mathrm{H}-3,110-113$ & 18.40 & N. truempyi & $<150$ & 0.78 & 0.83 & \\
\hline $3 \mathrm{H}-3,140-143$ & 18.70 & N. truempyi & $<150$ & 0.71 & 0.81 & \\
\hline $3 \mathrm{H}-4,0-3$ & 18.80 & N. truempyi & $<150$ & 0.79 & 0.81 & \\
\hline $3 \mathrm{H}-4,10-13$ & 18.90 & N. truempyi & $<150$ & 0.56 & 0.65 & \\
\hline $3 \mathrm{H}-4,20-23$ & 19.00 & N. truempyi & $<150$ & 0.80 & 0.73 & \\
\hline $3 \mathrm{H}-4,30-33$ & 19.10 & N. truempyi & $<150$ & 0.68 & 0.65 & \\
\hline $3 \mathrm{H}-4,40-43$ & 19.20 & N. truempyi & $<150$ & 0.88 & 0.83 & \\
\hline $3 \mathrm{H}-4,50-53$ & 19.30 & N. truempyi & $<150$ & 0.76 & 0.75 & \\
\hline $3 \mathrm{H}-4,60-63$ & 19.40 & N. truempyi & $<150$ & 0.01 & 0.34 & \\
\hline $3 \mathrm{H}-4,80-83$ & 19.60 & N. truempyi & $<150$ & 0.92 & 0.86 & \\
\hline $3 \mathrm{H}-4,90-93$ & 19.70 & N. truempyi & $<150$ & 0.88 & 0.85 & \\
\hline $3 \mathrm{H}-4,100-103$ & 19.80 & N. truempyi & $<150$ & 0.77 & 0.81 & \\
\hline $3 \mathrm{H}-4,110-113$ & 19.90 & N. truempyi & $<150$ & 1.11 & 0.96 & \\
\hline $3 \mathrm{H}-4,130-133$ & 20.10 & N. truempyi & $<150$ & 0.72 & 0.71 & \\
\hline $3 \mathrm{H}-4,140-143$ & 20.20 & N. truempyi & $<150$ & 0.64 & 0.64 & \\
\hline $3 \mathrm{H}-5,0-3$ & 20.30 & N. truempyi & $<150$ & 0.50 & 0.51 & \\
\hline $3 \mathrm{H}-5,0-3$ & 20.30 & N. truempyi & $<150$ & 0.50 & 0.51 & \\
\hline $3 \mathrm{H}-5,10-13$ & 20.40 & N. truempyi & $<150$ & 0.71 & 0.85 & \\
\hline $3 \mathrm{H}-5,10-13$ & 20.40 & N. truempyi & $<150$ & 0.71 & 0.85 & \\
\hline $3 \mathrm{H}-5,20-23$ & 20.50 & N. truempyi & $<150$ & 0.71 & 0.86 & \\
\hline $3 \mathrm{H}-5,30-33$ & 20.60 & N. truempyi & $<150$ & 0.76 & 0.83 & \\
\hline $3 \mathrm{H}-5,40-43$ & 20.70 & N. truempyi & $<150$ & 0.73 & 0.73 & \\
\hline $3 \mathrm{H}-5,60-63$ & 20.90 & N. truempyi & $<150$ & 0.68 & 0.76 & \\
\hline $3 \mathrm{H}-5,70-73$ & 21.00 & N. truempyi & $<150$ & 0.51 & 0.63 & \\
\hline $3 \mathrm{H}-5,80-83$ & 21.10 & N. truempyi & $<150$ & 0.68 & 0.70 & \\
\hline $3 \mathrm{H}-5,100-103$ & 21.30 & N. truempyi & $<150$ & 0.45 & 0.45 & \\
\hline $3 \mathrm{H}-5,110-113$ & 21.40 & N. truempyi & $<150$ & 0.49 & 0.57 & \\
\hline $3 \mathrm{H}-5,130-133$ & 21.60 & N. truempyi & $<150$ & 0.66 & 0.66 & \\
\hline $3 \mathrm{H}-6,10-12$ & 21.90 & N. truempyi & $<150$ & 0.65 & 0.75 & \\
\hline $3 \mathrm{H}-6,20-22$ & 22.00 & N. truempyi & $<150$ & 1.05 & 0.51 & \\
\hline $3 \mathrm{H}-6,30-32$ & 22.10 & N. truempyi & $<150$ & 0.77 & 0.74 & \\
\hline $3 \mathrm{H}-6,40-42$ & 22.20 & N. truempyi & $<150$ & 0.65 & 0.77 & \\
\hline $3 \mathrm{H}-6,50-52$ & 22.30 & N. truempyi & $<150$ & 1.36 & 1.08 & \\
\hline $3 \mathrm{H}-6,80-82$ & 22.60 & N. truempyi & $<150$ & 0.91 & 0.71 & \\
\hline $3 \mathrm{H}-6,100-102$ & 22.80 & N. truempyi & $<150$ & 0.95 & 0.94 & \\
\hline $3 \mathrm{H}-6,110-112$ & 22.90 & N. truempyi & $<150$ & 0.79 & 0.70 & \\
\hline $3 \mathrm{H}-6,120-122$ & 23.00 & N. truempyi & $<150$ & 0.79 & 0.76 & \\
\hline $3 \mathrm{H}-6,130-132$ & 23.10 & N. truempyi & $<150$ & 0.69 & 0.91 & \\
\hline $4 \mathrm{H}-1,60-62$ & 24.40 & N. truempyi & $<150$ & 0.67 & 0.93 & \\
\hline $4 \mathrm{H}-1,70-72$ & 24.50 & N. truempyi & $<150$ & 0.76 & 0.84 & \\
\hline $4 \mathrm{H}-1,80-82$ & 24.60 & N. truempyi & $<150$ & 0.80 & 0.90 & \\
\hline $4 \mathrm{H}-1,90-92$ & 24.70 & N. truempyi & $<150$ & 0.66 & 0.83 & \\
\hline $4 \mathrm{H}-1,100-102$ & 24.80 & N. truempyi & $<150$ & 0.74 & 0.84 & \\
\hline $4 \mathrm{H}-1,110-112$ & 24.90 & N. truempyi & $<150$ & 0.58 & 0.74 & \\
\hline $4 \mathrm{H}-1,130-132$ & 25.10 & N. truempyi & $<150$ & 0.60 & 0.76 & \\
\hline $4 \mathrm{H}-2,10-12$ & 25.40 & N. truempyi & $<150$ & 0.65 & 0.89 & \\
\hline $4 \mathrm{H}-2,20-22$ & 25.50 & N. truempyi & $<150$ & 0.54 & 0.20 & \\
\hline $4 \mathrm{H}-2,30-32$ & 25.60 & N. truempyi & $<150$ & 0.82 & 0.94 & \\
\hline $4 \mathrm{H}-2,50-52$ & 25.80 & N. truempyi & $<150$ & 0.64 & 0.85 & \\
\hline $4 \mathrm{H}-2,70-72$ & 26.00 & N. truempyi & $<150$ & 0.75 & 0.87 & \\
\hline $4 \mathrm{H}-2,80-82$ & 26.10 & N. truempyi & $<150$ & 0.55 & 0.33 & \\
\hline $4 \mathrm{H}-2,90-92$ & 26.20 & N. truempyi & $<150$ & 0.64 & 0.82 & \\
\hline $4 \mathrm{H}-2,100-102$ & 26.30 & N. truempyi & $<150$ & 0.58 & 0.48 & \\
\hline $4 \mathrm{H}-2,110-112$ & 26.40 & N. truempyi & $<150$ & 0.69 & 0.92 & \\
\hline $4 \mathrm{H}-2,130-132$ & 26.60 & N. truempyi & $<150$ & 0.67 & 0.83 & \\
\hline $4 \mathrm{H}-3,0-2$ & 26.80 & N. truempyi & $<150$ & 0.62 & 0.84 & \\
\hline $4 \mathrm{H}-3,10-12$ & 26.90 & N. truempyi & $<150$ & 0.46 & 0.79 & \\
\hline
\end{tabular}


B.S. WADE ET AL.

Data Report: Isotope Stratigraphy, LAte Middle Eocene

Table T1 (continued).

\begin{tabular}{|c|c|c|c|c|c|c|}
\hline $\begin{array}{l}\text { Core, section, } \\
\text { interval }(\mathrm{cm})\end{array}$ & $\begin{array}{l}\text { Depth } \\
\text { (mbsf) }\end{array}$ & Taxon & $\begin{array}{l}\text { Size } \\
(\mu \mathrm{m})\end{array}$ & $\delta^{18} \mathrm{O}$ & $\delta^{13} \mathrm{C}$ & $N$ \\
\hline $4 \mathrm{H}-3,20-22$ & 27.00 & N. truempyi & $<150$ & 0.61 & 0.60 & \\
\hline $4 \mathrm{H}-3,30-32$ & 27.10 & N. truempyi & $<150$ & 0.76 & 0.80 & \\
\hline $4 \mathrm{H}-3,40-42$ & 27.20 & N. truempyi & $<150$ & 0.63 & 0.74 & \\
\hline $4 \mathrm{H}-3,50-52$ & 27.30 & N. truempyi & $<150$ & 0.70 & 0.81 & \\
\hline $4 \mathrm{H}-3,60-62$ & 27.40 & N. truempyi & $<150$ & 0.75 & 0.77 & \\
\hline $4 \mathrm{H}-3,70-72$ & 27.50 & N. truempyi & $<150$ & 0.60 & 0.78 & \\
\hline $4 \mathrm{H}-3,80-82$ & 27.60 & N. truempyi & $<150$ & 0.57 & 0.81 & \\
\hline
\end{tabular}

Note: Blank spaces $=$ no measurement. 